\title{
Journal of real-time image processing: second issue of volume 6
}

\author{
Nasser Kehtarnavaz $\cdot$ Matthias F. Carlsohn
}

Published online: 2 March 2011

(C) Springer-Verlag 2011

We begin this issue by good news. We are pleased to inform you that JRTIP has been included in Thomson Reuters Science Citation Index. This marks a significant milestone in establishing JRTIP as a major source of reference in the area of real-time image and video processing. It is expected that this well-deserved recognition of JRTIP will lead to additional paper submissions.

The editorial board of JRTIP met in San Francisco in January of this year, where they were attending the SPIE Conference on Real-Time Image and Video Processing. A picture of some of the editorial board members present at the Springer sponsored dinner meeting is shown below. In this meeting, various issues related to the operation of JRTIP were discussed. Also, a number of solutions were presented in order to lower the average review time of papers.

The editorial board will have its next meeting in Brussels in April 2012, where they will attend the SPIE Conference on Real-Time Image and Video Processing as part of the SPIE Photonics Europe Symposium. The call for papers for this conference can be found at http://spie. org/photonics-europe.xml. We encourage submission of papers to this conference which is solely dedicated to real-time image and video processing research, and development.

This regular issue includes six papers covering algorithmic and software aspects of real-time image and video

\footnotetext{
N. Kehtarnavaz

Department of Electrical Engineering, University

of Texas at Dallas, Dallas, USA

M. F. Carlsohn ( $\square)$

Computer Vision \& Image Communication Engineering

and Consultancy Dr. Carlsohn, Bremen, Germany

e-mail: matthias.carlsohn@t-online.de
}

processing to enable real-time throughput with acceptable or tolerable loss in performance.

The first paper by Hosny et al. presents a fast, accurate, and memory-efficient method for computing orthogonal Fourier-Mellin moments. A comparison to conventional methods is provided indicating the efficiency of the proposed method.

The second paper by Spiliotis et al. also addresses the computation of moments that are widely used in image analysis and pattern recognition. An efficient computation approach is proposed by decomposing a gray level image into a set of binary images. The computational complexity is indicated to be $O\left(k L^{2}\right)$, where $k$ denotes number of blocks used and $L$ the moment order.

The third paper by Karunakar et al. proposes a technique to interactively extract region-of-interest (ROI) in Joint Video Team (JVT) scalable video coding. The proposed Interactive ROI (IROI) technique provides an enhancement of scalability which makes it suited for video decoding in wireless environment with limited bandwidth availability.

The fourth paper by Tan et al. covers a perceptually tuned sub-band image coder which is capable of selectively operating on the sub-bands of an image. The results obtained indicate that the use of this coder leads to a reduction in the computational cost without introducing perceptible distortion.

The fifth paper by Lacassagne et al. introduces two fast algorithms for connected component labeling in binary images. A comparison is made with an existing fast algorithm which is used for the same purpose. It is shown that these algorithms provide greater data independency and higher runtime predictability.

The sixth paper by Razlighi et al. introduces four computationally efficient methods for estimation of Image Spatial Entopy (ISE). ISE is increasingly being used in 
image processing applications. It is shown that by tolerating merely $1 \%$ estimation error, the order of complexity is significantly reduced.

As always, we thank all of the Associate Editors and reviewers for their ongoing efforts and contributions. We also wish to express our gratitude to the Guest Editors of several upcoming special issues. We would like to welcome to the JRTIP Editorial Board the following new Associate Editors who have acted as the Guest Editors of the past special issues: Dr. Barak Fishbain, Dr. Ruby Mehrubeoglu, Dr. João Pereira, Dr. Laurent Perroton, Dr. André Stork, and Dr. Sen Wang.

At the end, it is with great sorrow, we mention that Dr. Pierre Graebling, an Associate Editor of JRTIP, passed away in January after a long and exhaustive battle to recover his health. We and the Editorial Office staff are deeply saddened and express our condolences to his family and friends for their loss. In appreciation of his contributions to the Journal, we dedicate this issue to his memory and service to JRTIP.

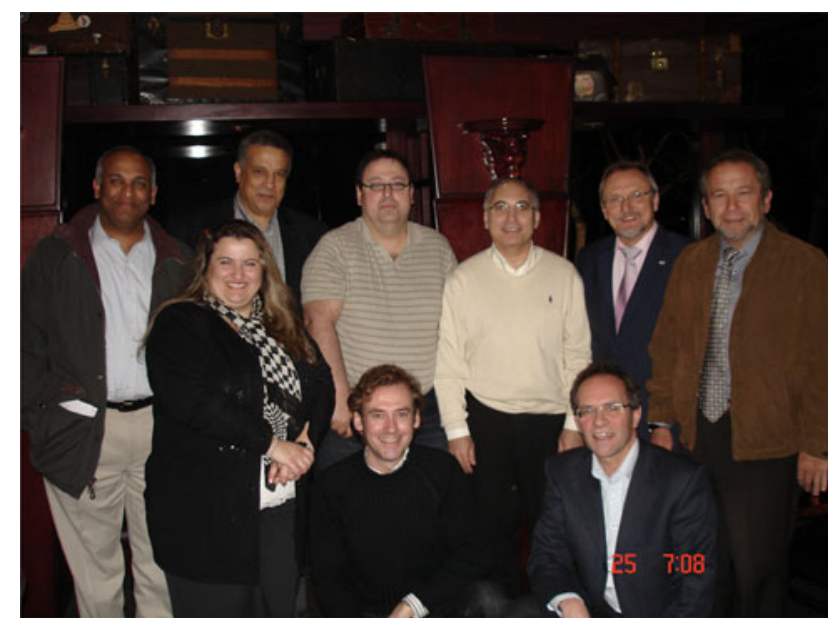

Left to right-(standing) Mukul Shirvaikar, Rube Mehrubeoglu, Mohamed Akil, Christos Grecos, Nasser Kehtarnavaz, Matthias Carlsohn, Volodymyr Ponomaryov, (sitting) Luis Salgado, Reinhard Koch 